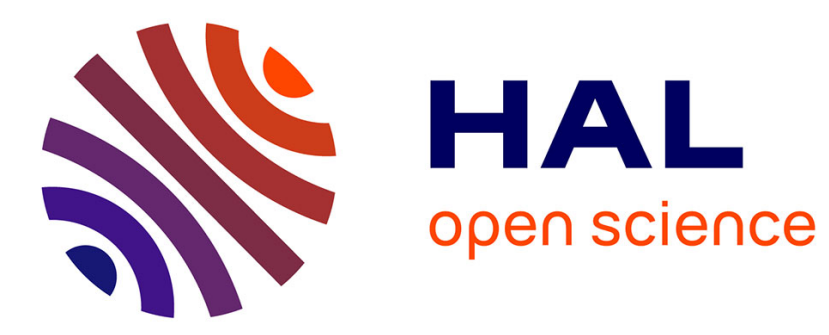

\title{
An Embedded Split-Step method for solving the nonlinear Schrodinger equation in optics
}

\author{
Stéphane Balac, Fabrice Mahé
}

\section{To cite this version:}

Stéphane Balac, Fabrice Mahé. An Embedded Split-Step method for solving the nonlinear Schrodinger equation in optics. Journal of Computational Physics, 2015, 280, pp.295-305. 10.1016/j.jcp.2014.09.018 . hal-00921656v2

\section{HAL Id: hal-00921656 \\ https://hal.science/hal-00921656v2}

Submitted on 14 Oct 2014

HAL is a multi-disciplinary open access archive for the deposit and dissemination of scientific research documents, whether they are published or not. The documents may come from teaching and research institutions in France or abroad, or from public or private research centers.
L'archive ouverte pluridisciplinaire HAL, est destinée au dépôt et à la diffusion de documents scientifiques de niveau recherche, publiés ou non, émanant des établissements d'enseignement et de recherche français ou étrangers, des laboratoires publics ou privés. 


\title{
An Embedded Split-Step method for solving the nonlinear Schrödinger equation in optics
}

\author{
Stéphane Balac ${ }^{\mathrm{a}, \mathrm{b}, *}$, Fabrice Mahéa,c \\ ${ }^{a}$ UEB, Université Européenne de Bretagne, Université de Rennes 1, France \\ ${ }^{b}$ CNRS UMR 6082 FOTON, Enssat, 6 rue de Kerampont, CS 80518, 22305 Lannion, France \\ ${ }^{c}$ IRMAR, Université de Rennes I, CNRS, Campus de Beaulieu, 35042 Rennes, France
}

\begin{abstract}
In optics the nonlinear Schrödinger equation (NLSE) which modelizes wave propagation in an optical fiber is mostly solved by the Symmetric Split-Step method. The practical efficiency of the Symmetric Split-Step method is highly dependent on the computational grid points distribution along the fiber, therefore an efficient adaptive step-size control strategy is mandatory. The most common approach for step-size control is the "step-doubling" approach. It provides an estimation of the local error for an extra computational cost of around $50 \%$. Alternatively there exist in optics literature other approaches based on the observation along the propagation length of the behavior of a given optical quantity. The step-size at each computational step is set so as to guarantee that the known properties of the quantity are preserved. These approaches derived under specific physical assumptions are low cost but suffer from a lack of generality. In this paper we present a new method for estimating the local error in the Symmetric Split-Step method when solving the NLSE. It conciliates the advantages of the step-doubling approach in terms of generality without the drawback of requiring a significant extra computational cost. The method is related to Embedded Split-Step methods for nonlinear evolution problems.
\end{abstract}

Keywords: Symmetric Split-Step method, adaptive step-size control, nonlinear Schrödinger Equation

2000 MSC: 35Q55, 35Q60, 65M99

\section{Introduction}

The nonlinear Schrõdinger equation (NLSE) describes a wide class of physical phenomena among which is propagation of light in an optical fiber. We are concerned by the following form of the NLSE (see $[1,2]$ )

$$
\frac{\partial}{\partial z} A(z, t)=-\frac{\alpha}{2} A(z, t)+\left(\sum_{n=2}^{N} \mathrm{i}^{n+1} \frac{\beta_{n}}{n !} \frac{\partial^{n}}{\partial t^{n}} A(z, t)\right)+\mathrm{i} \gamma A(z, t)|A(z, t)|^{2}
$$

\footnotetext{
${ }^{*}$ Corresponding author.

Email addresses: stephane.balac@univ-rennes1.fr (Stéphane Balac), fabrice.mahe@univ-rennes1.fr (Fabrice Mahé) 
where the complex valued function $A$ represents the slowly varying pulse envelope of a quasimonochromatic optical wave at frequency $\omega_{0}$ in a frame of reference moving with the pulse at the group velocity $v_{g}=c / n_{g}$ where $n_{g}$ denotes the group index of the fiber, $z$ represents the position along the fiber and $t$ the time in the local frame. In the situation considered here, the optical wave is represented by an electric field $\mathbf{E}$ at frequency $\omega_{0}$ which is linearly polarized along the vector $\mathbf{e}_{\mathbf{x}}$ transverse to the propagation's direction $\mathbf{e}_{\mathbf{z}}$ defined by the fiber axis and expressed as a function of position $\mathbf{r}=(x, y, z)$ and time $\tau$ in the form

$$
\mathbf{E}(\mathbf{r}, \tau)=A(z, t) F(x, y) \mathrm{e}^{-\mathrm{i}\left(\omega_{0} \tau-k z\right)} \mathbf{e}_{\mathbf{x}}
$$

where $F(x, y)$ is the electric wave transverse representation also called the "modal distribution" and $k$ is the wavenumber. The relation between the "local time" $t$ in the local frame and the absolute time $\tau$ is $t=\tau-z / v_{g}$.

Equation (1) describes wave propagation in a single mode fiber taking into account phenomena such as the optical Kerr effect through the nonlinear coefficient $\gamma$ and linear dispersion through the dispersion coefficients $\beta_{n}, n=2, \ldots, N$ with $N \in \mathbb{N}, N \geq 2$. The coefficient $\alpha$ accounts for attenuation or gain during propagation in the fiber. The partial differential equation (PDE) (1) is to be solved for all $z$ in a given interval $[0, L]$ where $L$ denotes the length of the fiber and for all "local time" $t \in \mathbb{R}$. It is considered together with the following boundary condition at $z=0: \forall t \in \mathbb{R}, A(0, t)=a_{0}(t)$, where $a_{0}$ is a given complex valued function.

A numerical method widely used for solving the NLSE in optics is the Symmetric SplitStep method, see e.g. [3-7], due to its particular simplicity and efficiency. The Symmetric Split-Step method applied to the NLSE (1) amounts to decompose over each subinterval of a given subdivision of the fiber length the PDE problem into a sequence of 3 simpler problems connected to each other. The first one corresponding to a purely linear PDE over the first half of the subinterval, the second one over the whole subinterval corresponding to a nonlinear ordinary differential equation (ODE) with the time variable as a parameter and the third one a purely linear PDE over the second half of the subinterval. The interest of this numerical approach for computing an approximation of the solution to the NLSE (1) is that each of the 3 nested problems can be solved more easily than equation (1) and when the step-size tends toward 0 the approximate solution is likely to converge to the solution of the NLSE (1) [8-10].

Let us now introduce a comprehensive mathematical framework for the study of our Embedded Split-Step method applied to the NLSE. We denote by $\mathbb{L}^{p}(I, \mathbb{C}), p \in[1,+\infty[$ the set of complex-valued functions over the real interval $I$ whose $p$-th powers are integrable and by $\mathbb{H}^{m}(I, \mathbb{C})$ for $m \in \mathbb{N}^{*}$ the Sobolev space of functions in $\mathbb{L}^{2}(I, \mathbb{C})$ with derivatives up to order $m$ in $\mathbb{L}^{2}(I, \mathbb{C})$, see e.g. [11]. For convenience, we will also use the notation $\mathbb{H}^{0}(I, \mathbb{C})$ for $\mathbb{L}^{2}(I, \mathbb{C})$. The Sobolev spaces $\mathbb{H}^{m}(I, \mathbb{C}), m \in \mathbb{N}$, are equipped with the usual norms denoted \|\|$_{m}$. For a function $A:(z, t) \in \mathbb{R}^{2} \mapsto A(z, t) \in \mathbb{C}$, we denote by $A(z)$ the first partial function of $A$ in $z$, i.e. $A(z): t \in \mathbb{R} \mapsto A(z, t) \in \mathbb{C}$. The NLSE (1) can be reformulated as

$$
\frac{\partial}{\partial z} A(z)=\mathcal{D} A(z)+\mathcal{N}(A(z)) \quad \forall z \in[0, L]
$$

where the linear operator $\mathcal{D}$ given by

$$
\mathcal{D}: A(z) \longmapsto \sum_{n=2}^{N} \beta_{n} \frac{\mathrm{i}^{n+1}}{n !} \partial_{t}^{n} A(z)
$$


is a unbounded linear operator on $\mathbb{L}^{2}(\mathbb{R}, \mathbb{C})$ with domain $\mathbb{H}^{N}(\mathbb{R}, \mathbb{C})$ and the non-linear operator $\mathcal{N}$ given by

$$
\mathcal{N}: A(z) \in \mathbb{L}^{6}(\mathbb{R}, \mathbb{C}) \longmapsto-\frac{1}{2} \alpha A(z)+\mathrm{i} \gamma A(z)|A(z)|^{2} \in \mathbb{L}^{2}(\mathbb{R}, \mathbb{C})
$$

is locally Lipschitz continuous on every Sobolev space $\mathbb{H}^{m}(\mathbb{R}, \mathbb{C})$ for all $m \in \mathbb{N}^{*}$ with additionally $\mathcal{N} \in \mathbb{C}^{\infty}\left(\mathbb{H}^{m}(\mathbb{R}, \mathbb{C}), \mathbb{H}^{m}(\mathbb{R}, \mathbb{C})\right)$, see [12]. Another splitting for the NLSE (1) where the term $-\frac{1}{2} \alpha A$ is added to the linear operator $\mathcal{D}$ instead of the nonlinear operator $\mathcal{N}$ is also possible.

For all $(k, m) \in \mathbb{N}^{2}$ and $I \subset \mathbb{R}$, we denote by $C^{k}\left(I ; \mathbb{H}^{m}(\mathbb{R}, \mathbb{C})\right)$ the space of functions $u: z \in I \mapsto u(z) \in \mathbb{H}^{m}(\mathbb{R}, \mathbb{C}$ ) with continuous derivatives up to order $k$ (or just continuous when $k=0$ ). When $N=2$, a comprehensive mathematical framework for the NLSE (1) exists in the literature [13] and it is known that for $a_{0} \in \mathbb{H}^{2}(\mathbb{R}, \mathbb{C})$ there exists a unique $A$ belonging to $C^{0}\left(\mathbb{R} ; \mathbb{H}^{2}(\mathbb{R}, \mathbb{C})\right) \cap C^{1}\left(\mathbb{R} ; \mathbb{L}^{2}(\mathbb{R}, \mathbb{C})\right)$ solution of equation (1) satisfying $A(0)=a_{0}$. This result has been extended to an arbitrary value of $N$ in [14]. Namely, denoting for any $m \in \mathbb{N}$ by $E_{m, N}(I)$ the space $\bigcap_{k=0}^{\lfloor m / N\rfloor} C^{k}\left(I, \mathbb{H}^{m-N k}(\mathbb{R}, \mathbb{C})\right)$, where $\lfloor s\rfloor$ denotes the integer part of $s \in \mathbb{R}^{+}$, the following result holds [14].

Theorem 1.1. For all $a_{0} \in \mathbb{H}^{m}(\mathbb{R}, \mathbb{C})$, with $m \in \mathbb{N}^{*}$, there exists a unique maximal solution $A \in E_{m, N}([0, Z[), Z \in] 0,+\infty]$, to the NLSE (1) with the initial condition $A(0)=a_{0}$ at $z=0$. This solution satisfies

$$
\|A(z)\|_{0}=\mathrm{e}^{-\frac{\alpha}{2} z}\left\|a_{0}\right\|_{0} \quad \text { for all } z \in[0, Z[.
$$

Moreover, if $N$ is even and $m \geq N / 2$ then the solution is global, i.e. $Z=+\infty$.

As a corollary of theorem 1.1 we have that when $N$ is an even integer and $a_{0} \in \mathbb{H}^{N}(\mathbb{R}, \mathbb{C})$ the NLSE (1) with the initial condition $A(0)=a_{0}$ at $z=0$ has a unique solution in the space $C^{0}\left([0, L] ; \mathbb{H}^{N}(\mathbb{R}, \mathbb{C})\right) \cap C^{1}\left([0, L] ; \mathbb{L}^{2}(\mathbb{R}, \mathbb{C})\right)$.

Of course, the practical efficiency of a numerical method such as the Symmetric Split-Step method applied to the NLSE (1) highly depends on the distribution of the computational grid points along the fiber and the use of an adaptive step-size control strategy is mandatory. The idea behind an adaptive step-size strategy is to introduce the grid points during the progress of the computation taking into account the information available at the current computation stage in order to determine the best suited step size (and therefore the next grid point) so as to maintain a given predefined accuracy of the approximation. Since it is not possible in practice to know the global discretization error, the step-size is determined so that a "local error" is lower than a prescribed tolerance. In the literature dedicated to optics, a variety of adaptive step-size strategies have been proposed to be used in conjunction with Split-Step methods for solving the NLSE (1). We can distinguish 2 types of approaches. The one based on physical concepts (or physical intuition) where at each grid point the step-size is chosen so as a "local error" related quantity estimated from a physical quantity matches the prescribed tolerance value. For instance, in the so-called "nonlinear phase rotation method" [4] the step-size is chosen so that the phase change due to nonlinearity does not exceed a certain limit. In the "walk-off" method, the step-size is chosen to be inversely proportional to the product of the absolute value of the dispersion and the spectral bandwidth of the signal and the method applies to low power, multichannel systems [4]. In $[15,16]$ a method termed the "uncertainty principle method" is proposed where the determination of the step-size is done from the values of a parameter derived from an inequality which in quantum mechanics gives rise to the uncertainty principle between two non-commuting operators. In [17] it is made use of the conservation of the "optical photon number" to estimate a local 
error related quantity and to define an adaptive step-size control strategy termed the Conservation Quantity Error method. This method applies to low loss fibers.

Another approach for defining adaptive step-size strategies consists in using purely numerical concepts. The "local error" is then defined as the error made using the numerical scheme when computing an approximation of the solution at the current grid point under the assumption that the value at the previous grid point was exact. In [4] the authors propose to use the "step-doubling" concept to estimate the local error when solving the NLSE by the Symmetric Split-Step method. This approach is closely related to the Step-Doubling method for local error estimation when numerically solving ordinary differential equations [18-20]. The main advantage of the Step-Doubling method compared to the previous ones is its generality since no assumption on the physical parameters involved in the equation is made. However a drawback of the Step-Doubling method is a computational over-cost of approximatively $50 \%$ (when compared to the same Split-Step method with the "optimal" grid points distribution given in advance).

In this paper we propose another way of estimating the local error in the Symmetric SplitStep method. The over-cost of the propound method is low. As for the Step-Doubling method, 2 approximate solutions of the NLSE corresponding to a "fine" solution and to a "coarse" solution are combined in a specific way to deliver a local error estimate. However, whereas in the StepDoubling method the 2 approximate solutions are obtained by the use of the Symmetric SplitStep method on 2 mesh grids, the finest one having twice the number of grid points of the coarse one, our method uses the same mesh grid but 2 Split-Step schemes of different order in the spirit of embedded Runge-Kutta methods for ordinary differential equation [19, 20]: we use the Symmetric Split-Step scheme which is known to have second order of accuracy with a dedicated first order of accuracy Split-Step scheme. In order to reduce the over-cost for estimating the local error, the idea is to re-use some of the computations required by the Symmetric SplitStep scheme when computing the coarse solution by the first order Split-Step scheme. The first order Split-Step scheme is therefore non-conventional and has been designed to meet this goal. The method is related to the embedded Split-Step formulae for the time integration of nonlinear evolution equation recently presented in [21] where the authors introduce pairs of related splitstep time integration formulae for the numerical solution of evolution equations. In particular they propose a trivial embedded split-step pair based on the second-order Strang splitting and the first-order Lie-Trotter splitting methods and a split-step pair of order 4(3) based on a fourthorder Runge-Kutta-Nystrom type method. These embedded split-step pairs are constructed so that some of the computations, corresponding to the main computational task in the propound examples, coincide. When dealing with the nonlinear Schrödinger equation in a two-dimensional domain or with the reaction-diffusion equation in a three-dimensional domain as in [21], the main computational task in the Split-Step method is the numerical approximation of the linear part of the equation realized by pseudo-spectral methods, whereas the numerical approximation of the nonlinear part involves cheap point-wise multiplications. When dealing with the NLSE (1), which is set in a one-dimensional domain, both the linear and nonlinear parts of the equation require approximately the same computational effort and the split-step pairs formulae proposed in [21] are not well adapted in this situation (the 2 split-step formulae do not share enough computational stages and the additional computational effort to estimate the local error becomes substantial). On the contrary, the split-step pair of order 2(1) based on the second-order Strang splitting formula derived in this paper for the NLSE (1) yields estimates of the local error for adaptive step-size purposes with a very low additional computational effort since the substeps of the Strang splitting formula are fully reused to compute the solution of the first order splitting 
formula.

The paper is organized as follows. To gain clarity we recall in section 2 the main features of the Symmetric Split-Step method based on Strang formula for solving the NLSE (1). In Section 3 we present a first order Split-Step scheme embedded in the Symmetric Split-Step scheme designed to deliver a local error estimate at a very cheap cost. In Section 4 the algorithm of our Embedded Split-Step method and the underlying step-size control strategy are detailed. Finally, in Section 5 we achieve a numerical comparison of our method with the Step-Doubling method [4] and the second-order Strang splitting method with first-order Lie-Trotter embedded splitting propound in [21] on benchmark problems in optics.

\section{The Symmetric Split-Step method applied to the NLSE}

In the Symmetric Split-Step scheme applied to the NLSE (1), the interval $[0, L]$ is divided into $K$ subintervals where the spatial grid points are denoted $z_{k}, k \in\{0, \ldots, K\}$, where $0=z_{0}<$ $z_{1}<\cdots<z_{K-1}<z_{K}=L$. We also denote by $h_{k}=z_{k+1}-z_{k}$ the step-size between grid points $z_{k}$ and $z_{k+1}$ and we set $z_{k+\frac{1}{2}}=z_{k}+\frac{h_{k}}{2}$. The Symmetric Split-Step method applied to the NLSE (1) amounts to solve over each subinterval $\left[z_{k}, z_{k+1}\right]$ for $k \in\{0, \ldots, K-1\}$, the following 3 nested problems with time variable $t$ as a parameter and the operators $\mathcal{D}$ and $\mathcal{N}$ defined as in (4) and (5):

$$
\left\{\begin{aligned}
\frac{\partial}{\partial z} A_{k}^{+}(z) & =\mathcal{D} A_{k}^{+}(z) \quad \forall z \in\left[z_{k}, z_{k+\frac{1}{2}}\right] \\
A_{k}^{+}\left(z_{k}\right) & =A_{k-1}^{[2]}\left(z_{k}\right)
\end{aligned}\right.
$$

where $A_{k-1}^{[2]}\left(z_{k}\right)$ represents the approximate solution at grid point $z_{k}$ computed by the Symmetric Split-Step method at step $k-1$;

$$
\left\{\begin{aligned}
\frac{\partial}{\partial z} B_{k}(z) & =\mathcal{N}\left(B_{k}(z)\right) \quad \forall z \in\left[z_{k}, z_{k+1}\right] \\
B_{k}\left(z_{k}\right) & =A_{k}^{+}\left(z_{k+\frac{1}{2}}\right)
\end{aligned}\right.
$$

where $A_{k}^{+}\left(z_{k+\frac{1}{2}}\right)$ represents the solution to problem (7) at half grid point $z_{k+\frac{1}{2}}$;

$$
\left\{\begin{array}{l}
\frac{\partial}{\partial z} A_{k}^{-}(z)=\mathcal{D} A_{k}^{-}(z) \quad \forall z \in\left[z_{k+\frac{1}{2}}, z_{k+1}\right] \\
A_{k}^{-}\left(z_{k+\frac{1}{2}}\right)=B_{k}\left(z_{k+1}\right)
\end{array}\right.
$$

where $B_{k}\left(z_{k+1}\right)$ represents the solution to problem (8) at grid point $z_{k+1}$. An approximate solution to the NLSE (1) at grid point $z_{k+1}$ is then given by $A_{k}^{[2]}\left(z_{k+1}\right)=A_{k}^{-}\left(z_{k+1}\right)$. The exponent ${ }^{[2]}$ in the notation of the approximate solution refers to the second order of accuracy of the Symmetric Split-Step method. In the splitting figured out by the 3 connected problems (7), (8) and (9) the role of the linear operator $\mathcal{D}$ and nonlinear operator $\mathcal{N}$ can be interchanged. In general the choice is guided by a comparison of the cost for solving numerically the linear and nonlinear problems: the more expensive problem should be placed in the middle to be solved only one time per step. In the special case of the NLSE (1) the 2 formulations are roughly identical from a computational point of view. 
The solution to problems (7) and (9) can be computed by using the Fourier Transform (FT) approach. The solution to problem (7) at grid point $z_{k+\frac{1}{2}}$ reads

$$
A_{k}^{+}\left(z_{k+\frac{1}{2}}\right)=\mathcal{F}^{-1}\left[\widehat{A}_{k-1}\left(z_{k}\right) \mathrm{e}^{\widehat{d}_{v} \frac{h_{k}}{2}}\right]
$$

and the solution to problem (9) at grid point $z_{k+1}$ reads

$$
A_{k}^{-}\left(z_{k+1}\right)=\mathcal{F}^{-1}\left[\widehat{B}_{k}\left(z_{k+1}\right) \mathrm{e}^{\widehat{d}_{v} \frac{h_{k}}{2}}\right]
$$

where $\widehat{A}_{k-1}$ and $\widehat{B}_{k}$ denote the Fourier Transform of $A_{k-1}$ and $B_{k}$ respectively, $\mathcal{F}^{-1}$ the inverse Fourier operator and

$$
\widehat{d_{v}}=\mathrm{i} \sum_{n=2}^{N} \frac{\beta_{n}}{n !}(2 \pi v)^{n} .
$$

The solution to problem (8) when $\mathcal{N}$ is given by (5) can be computed analytically. At grid point $z_{k+1}$ we have

$$
B_{k}\left(z_{k+1}\right)=\left\{\begin{array}{ll}
A_{k}^{+}\left(z_{k+\frac{1}{2}}\right) \exp \left(\mathrm{i} \gamma h_{k}\left|A_{k}^{+}\left(z_{k+\frac{1}{2}}\right)\right|^{2}\right) & \text { if } \alpha=0 \\
A_{k}^{+}\left(z_{k+\frac{1}{2}}\right) \exp \left(-\frac{\alpha}{2} h_{k}-\frac{\mathrm{i} \gamma}{\alpha}\left|A_{k}^{+}\left(z_{k+\frac{1}{2}}\right)\right|^{2}\left(\mathrm{e}^{-\alpha h_{k}}-1\right)\right) & \text { if } \alpha \neq 0
\end{array} .\right.
$$

The convergence of Split-Step methods applied to various forms of the Schrödinger equation is widely documented in the literature in the case when $N=2$, see e.g. [8-10] where the authors prove that the convergence order of the Symmetric Split-Step method is 2. In the situation considered here the following result holds (see Proposition 3.1 of [14]).

Proposition 2.1. Let $A$ denote the solution to the NLSE (1) and for all $k \in\{0, \ldots, K-1\}$ let $A_{k}^{[2]}\left(z_{k+1}\right)$ denote its approximation at grid point $z_{k+1}$ computed by solving the 3 nested problems (7)-(8)-(9) with the initial condition $A_{k-1}^{[2]}\left(z_{k}\right)=A\left(z_{k}\right)$ assumed to be in $\mathbb{H}^{2 N+1}(\mathbb{R}, \mathbb{C})$. Then, for $h_{k}$ in a neighborhood of 0 we have the following estimate in $\mathbb{H}^{1}(\mathbb{R}, \mathbb{C})$ :

$$
A\left(z_{k+1}\right)=A_{k}^{[2]}\left(z_{k+1}\right)+O\left(h_{k}^{3}\right) .
$$

\section{A truncated first order Split-Step scheme}

\subsection{First-order Split-Step schemes}

As propounded in [21], a local error estimate can be obtained by using the first order LieTrotter Split-Step scheme together with the Symmetric Split-Step one. The Lie-Trotter Split-Step method amounts to solve for all $k \in\{1, \ldots, K-1\}$ the following 2 connected PDE problems:

$$
\left\{\begin{aligned}
\frac{\partial}{\partial z} A_{k}^{*}(z) & =\mathcal{D} A_{k}^{*}(z) \quad \forall z \in\left[z_{k}, z_{k+1}\right] \\
A_{k}^{*}\left(z_{k}\right) & =A_{k-1}^{[1]}\left(z_{k}\right)
\end{aligned}\right.
$$

where $A_{k-1}^{[1]}\left(z_{k}\right)$ represents the approximate solution at grid point $z_{k}$ computed at step $k-1$; and

$$
\left\{\begin{aligned}
& \frac{\partial}{\partial z} B_{k}(z)=\mathcal{N}\left(B_{k}\right)(z) \quad \forall z \in\left[z_{k}, z_{k+1}\right] \\
& B_{k}\left(z_{k}\right)=A_{k}^{*}\left(z_{k+1}\right) \\
& 6
\end{aligned}\right.
$$


where $A_{k}^{*}\left(z_{k+1}\right)$ represents the solution to problem (14) at point $z_{k+1}$. The approximate solution to the NLSE (1) at grid point $z_{k+1}$ is then $A_{k}^{[1]}\left(z_{k+1}\right)=B_{k}\left(z_{k+1}\right)$. The main drawback in using the LieTrotter Split-Step scheme to design a embedded split-step pair is that the computations leading to the approximate solution cannot be factorized with the one of the Symmetric Split-Step scheme. The consequence is a significant extra cost for estimating the local error in this way, making the method not competitive when compared to the Step-Doubling method.

We propose another first-order Split-Step scheme where most of the computations required for evaluating the first order approximate solution is also required for evaluating the Symmetric Split-Step approximate solution. The overall cost of this method for evaluating the local error is therefore very low. Namely, we consider the following Split-Step scheme defined for all $k \in\{1, \ldots, K-1\}$ by:

$$
\left\{\begin{aligned}
\frac{\partial}{\partial z} A_{k}^{\star}(z) & =\mathcal{D} A_{k}^{\star}(z) \quad \forall z \in\left[z_{k}, z_{k+\frac{1}{2}}\right] \\
A_{k}^{\star}\left(z_{k}\right) & =A_{k-1}^{[1]}\left(z_{k}\right)
\end{aligned}\right.
$$

where $A_{k-1}^{[1]}\left(z_{k}\right)$ represents the approximate solution at grid point $z_{k}$ computed at step $k-1$; and

$$
\left\{\begin{aligned}
\frac{\partial}{\partial z} B_{k}(z) & =\mathcal{N}\left(B_{k}\right)(z) \quad \forall z \in\left[z_{k}, z_{k+1}\right] \\
B_{k}\left(z_{k}\right) & =A_{k}^{\star}\left(z_{k+\frac{1}{2}}\right)
\end{aligned}\right.
$$

where $A_{k}^{\star}\left(z_{k+\frac{1}{2}}\right)$ represents the solution to problem (16) at half grid point $z_{k+\frac{1}{2}}$. The approximate solution to equation (1) at grid point $z_{k+1}$ computed from the approximate solution $A_{k-1}^{[1]}\left(z_{k}\right)$ at grid point $z_{k}$ is obtained by adding to $B_{k}\left(z_{k+1}\right)$ a corrective term as follows:

$$
A_{k}^{[1]}\left(z_{k+1}\right)=B_{k}\left(z_{k+1}\right)+\frac{1}{2} h_{k} \mathcal{D} A_{k-1}^{[1]}\left(z_{k}\right) .
$$

The exponent ${ }^{[1]}$ in the notation of the approximate solution refers to the first order of accuracy of the method (this point is justified in the next section).

\subsection{Error analysis of the truncated Split-Step scheme}

Since we are concerned by an analysis of the local error in the truncated Split-Step scheme defined by (16)-(17)-(18), we will assume that at stage $k$ for all $k \in\{0, \ldots, K-1\}$, the initial data $A_{k-1}^{[1]}\left(z_{k}\right)$ is exact, i.e. $A_{k-1}^{[1]}\left(z_{k}\right)=A\left(z_{k}\right)$ where $A$ denotes the solution to equation (1).

Lemma 3.1. For all $k \in\{0, \ldots, K-1\}$, let $A_{k}^{\star}$ be the solution of problem (16) with the initial condition given by $A_{k}^{\star}\left(z_{k}\right)=A\left(z_{k}\right)$ assumed to belong to $\mathbb{H}^{2 N+1}(\mathbb{R}, \mathbb{C})$. For $h_{k} \in \mathbb{R}_{+}^{*}$ in a neighborhood of 0 we have the following equality in $\mathbb{H}^{1}(\mathbb{R}, \mathbb{C})$

$$
A_{k}^{\star}\left(z_{k+\frac{1}{2}}\right)=A\left(z_{k}\right)+\frac{1}{2} h_{k} \mathcal{D} A\left(z_{k}\right)+O\left(h_{k}^{2}\right) .
$$

Proof. A first order Taylor expansion for $A_{k}^{\star}$ between $z_{k}$ and $z_{k+\frac{1}{2}}$ gives in $\mathbb{H}^{1}(\mathbb{R}, \mathbb{C})$

$$
A_{k}^{\star}\left(z_{k+\frac{1}{2}}\right)=A_{k}^{\star}\left(z_{k}\right)+\frac{1}{2} h_{k} \frac{\partial}{\partial z} A_{k}^{\star}\left(z_{k}\right)+O\left(h_{k}^{2}\right) .
$$

Since $A_{k}^{\star}$ is solution to problem (16), we have $\frac{\partial}{\partial z} A_{k}^{\star}\left(z_{k}\right)=\mathcal{D} A_{k}^{\star}\left(z_{k}\right)$. Combining the 2 equalities gives the result under the assumption $A_{k}^{\star}\left(z_{k}\right)=A\left(z_{k}\right)$. 
Lemma 3.2. For all $k \in\{0, \ldots, K-1\}$, consider the 2 nested problems (16)- (17) with the initial condition for problem $(16)$ given by $A_{k}^{\star}\left(z_{k}\right)=A\left(z_{k}\right)$ assumed to belong to $\mathbb{H}^{2 N+1}(\mathbb{R}, \mathbb{C})$. For $h_{k} \in \mathbb{R}_{+}^{*}$ in a neighborhood of 0 , the solution $B_{k}$ of problem (17) satisfies the following equality in $\mathbb{H}^{1}(\mathbb{R}, \mathbb{C})$ :

$$
B_{k}\left(z_{k+1}\right)=A\left(z_{k+1}\right)-\frac{1}{2} h_{k} \mathcal{D} A\left(z_{k}\right)+O\left(h_{k}^{2}\right) .
$$

Proof. A first order Taylor expansion applied to the solution $B_{k}$ to problem (17) between $z_{k}$ and $z_{k+1}$ gives in $\mathbb{H}^{1}(\mathbb{R}, \mathbb{C})$ :

$$
\begin{aligned}
B_{k}\left(z_{k+1}\right) & =B_{k}\left(z_{k}\right)+h_{k} \frac{\partial}{\partial z} B_{k}\left(z_{k}\right)+O\left(h_{k}^{2}\right)=B_{k}\left(z_{k}\right)+h_{k} \mathcal{N}\left(B_{k}\right)\left(z_{k}\right)+O\left(h_{k}^{2}\right) \\
& =A_{k}^{\star}\left(z_{k+\frac{1}{2}}\right)+h_{k} \mathcal{N}\left(A_{k}^{\star}\right)\left(z_{k+\frac{1}{2}}\right)+O\left(h_{k}^{2}\right) .
\end{aligned}
$$

Let us then consider a first order Taylor expansion of the solution to the NLSE (1) between $z_{k}$ and $z_{k+1}$ :

$$
A\left(z_{k+1}\right)=A\left(z_{k}\right)+h_{k} \frac{\partial}{\partial z} A\left(z_{k}\right)+O\left(h_{k}^{2}\right)=A\left(z_{k}\right)+h_{k}\left(\mathcal{D} A\left(z_{k}\right)+\mathcal{N}(A)\left(z_{k}\right)\right)+O\left(h_{k}^{2}\right)
$$

It follows that

$$
\begin{aligned}
A\left(z_{k+1}\right)-B_{k}\left(z_{k+1}\right) & =\left(A\left(z_{k}\right)-A_{k}^{\star}\left(z_{k+\frac{1}{2}}\right)\right)+h_{k} \mathcal{D} A\left(z_{k}\right) \\
& +h_{k}\left(\mathcal{N}(A)\left(z_{k}\right)-\mathcal{N}\left(A_{k}^{\star}\right)\left(z_{k+\frac{1}{2}}\right)\right)+\mathcal{O}\left(h_{k}^{2}\right) .
\end{aligned}
$$

Now consider a Taylor expansion of the operator $\mathcal{N}$ between $A_{k}^{\star}\left(z_{k+\frac{1}{2}}\right)$ and $A\left(z_{k}\right)$ :

$$
\mathcal{N}(A)\left(z_{k}\right)-\mathcal{N}\left(A_{k}^{\star}\right)\left(z_{k+\frac{1}{2}}\right)=\mathcal{N}^{\prime}\left(A_{k}^{\star}\left(z_{k+\frac{1}{2}}\right)\right)\left(A\left(z_{k}\right)-A_{k}^{\star}\left(z_{k+\frac{1}{2}}\right)\right)+O\left(\left\|A\left(z_{k}\right)-A_{k}^{\star}\left(z_{k+\frac{1}{2}}\right)\right\|_{1}^{2}\right) .
$$

From Lemma 3.1 we deduce that $\mathcal{N}(A)\left(z_{k}\right)-\mathcal{N}\left(A_{k}^{\star}\right)\left(z_{k+\frac{1}{2}}\right)=O\left(h_{k}\right)$ so that from (20)

$$
A\left(z_{k+1}\right)-B_{k}\left(z_{k+1}\right)=\left(A\left(z_{k}\right)-A_{k}^{\star}\left(z_{k+\frac{1}{2}}\right)\right)+h_{k} \mathcal{D} A\left(z_{k}\right)+O\left(h_{k}^{2}\right) .
$$

From Lemma 3.1 again, we conclude that $A\left(z_{k+1}\right)-B_{k}\left(z_{k+1}\right)=\frac{1}{2} h_{k} \mathcal{D} A\left(z_{k}\right)+O\left(h_{k}^{2}\right)$.

From Lemmas 3.1 and 3.2 we finally deduce the following result which states that the SplitStep scheme (16)-(17)-(18) is first order accurate.

Proposition 3.3. Let $A$ denote the solution to the NLSE (1) under the initial condition $A(0)=a_{0}$ at $z=0$ where $a_{0}$ is a given function in $\mathbb{H}^{2 N+1}(\mathbb{R}, \mathbb{C})$. For all $k \in\{0, \ldots, K-1\}$, let $A_{k}^{[1]}\left(z_{k+1}\right)$ be the function given by relation (18) where $B_{k}\left(z_{k+1}\right)$ is the solution at grid point $z_{k+1}$ of the 2 nested problems (16)-(17) under the initial condition for problem (16) given by $A_{k}^{\star}\left(z_{k}\right)=A\left(z_{k}\right)$. For $h_{k} \in \mathbb{R}_{+}^{*}$ in a neighborhood of 0 we have the following equality in $\mathbb{H}^{1}(\mathbb{R}, \mathbb{C})$ :

$$
A\left(z_{k+1}\right)=A_{k}^{[1]}\left(z_{k+1}\right)+O\left(h_{k}^{2}\right) .
$$

Remark 1. A first order Taylor expansion of the solution to problem (9) between $z_{k+\frac{1}{2}}$ and $z_{k+1}$ gives

$$
A_{k}^{-}\left(z_{k+1}\right)=A_{k}^{-}\left(z_{k+\frac{1}{2}}\right)+\frac{h_{k}}{2} \frac{\partial}{\partial z} A_{k}^{-}\left(z_{k+\frac{1}{2}}\right)+O\left(h_{k}^{2}\right)=B_{k}\left(z_{k+1}\right)+\frac{h_{k}}{2} \mathcal{D} B_{k}\left(z_{k+1}\right)+O\left(h_{k}^{2}\right) .
$$


From Lemma 3.2 and from (19) we deduce successively that

$$
A_{k}^{[2]}\left(z_{k+1}\right)=A_{k}^{-}\left(z_{k+1}\right)=B_{k}\left(z_{k+1}\right)+\frac{h_{k}}{2} \mathcal{D} A\left(z_{k+1}\right)+O\left(h_{k}^{2}\right)=B_{k}\left(z_{k+1}\right)+\frac{h_{k}}{2} \mathcal{D} A\left(z_{k}\right)+O\left(h_{k}^{2}\right) .
$$

Therefore, under the assumption that the initial data $A_{k-1}^{[1]}\left(z_{k}\right)$ is exact, i.e. $A_{k-1}^{[1]}\left(z_{k}\right)=A\left(z_{k}\right)$, the corrective term (18) is nothing but a first order Taylor expansion of the solution to problem (9). A similar first order Taylor expansion could be considered instead of solving problem (7) with a resulting approximation scheme having the same order of accuracy, but the computational procedure would not be anymore fully embedded in the Symmetric Split-Step one, resulting in an increase of the computational cost.

Remark 2. The error estimates in Propositions 2.1 and 3.3 are set up in $\mathbb{H}^{1}(\mathbb{R}, \mathbb{C})$. Using advanced techniques developed by $\mathrm{C}$. Lubich [10] for $\mathbb{L}^{2}$-estimates of splitting integrators using $\mathbb{H}^{1}$-conditional stability, Proposition 2.1 can be formulated in $\mathbb{L}^{2}(\mathbb{R}, \mathbb{C})$, see [14]. We have every reason to think that the same techniques would allow to extend the error estimate of Proposition 3.3 to $\mathbb{L}^{2}(\mathbb{R}, \mathbb{C})$ but the proof of this statement is beyond the scope of this paper.

\section{The Embedded Split-Step method with adaptive step-size control}

\subsection{Numerical approximation of the local error for the Split-Step scheme}

Assuming that the solution value at grid point $z_{k}$ is regarded as exact (because we are concerned by an estimation of the local error), we denote by $A_{k}^{[1]}\left(\right.$ resp. $\left.A_{k}^{[2]}\right)$ the approximate solution computed at the current grid point $z_{k}$ by the first order (resp. the second order) above Split-Step schemes. From Propositions 2.1 and 3.3 we deduce that for a regular enough initial condition, the local error at grid point $z_{k+1}$ for each of the 2 schemes is respectively given in $\mathbb{H}^{1}(\mathbb{R}, \mathbb{C}$ ) (or in $\mathbb{L}^{2}(\mathbb{R}, \mathbb{C})$, see Remark 2 ), for $h_{k}$ in a neighborhood of 0 , by

$$
\begin{aligned}
& \ell_{k+1}^{[1]}=\left\|A\left(z_{k+1}\right)-A_{k}^{[1]}\left(z_{k+1}\right)\right\|=C_{1, k} h_{k}^{2}+O\left(h_{k}^{3}\right) \\
& \ell_{k+1}^{[2]}=\left\|A\left(z_{k+1}\right)-A_{k}^{[2]}\left(z_{k+1}\right)\right\|=C_{2, k} h_{k}^{3}+O\left(h_{k}^{4}\right)
\end{aligned}
$$

where $C_{1, k}$ and $C_{2, k}$ denote 2 numbers independent of $h_{k}$. Then,

$$
\left\|A_{k}^{[2]}\left(z_{k+1}\right)-A_{k}^{[1]}\left(z_{k+1}\right)\right\| \leq\left\|A\left(z_{k+1}\right)-A_{k}^{[2]}\left(z_{k+1}\right)\right\|+\left\|A\left(z_{k+1}\right)-A_{k}^{[1]}\left(z_{k+1}\right)\right\|=C_{1, k} h_{k}^{2}+O\left(h_{k}^{3}\right) .
$$

Thus the local error for the first order Split-Step scheme at grid point $z_{k+1}$ can be approximated, with an error in $O\left(h_{k}^{3}\right)$, in the following way:

$$
\ell_{k+1}^{[1]} \approx C_{1, k} h_{k}^{2} \approx\left\|A_{k}^{[2]}\left(z_{k+1}\right)-A_{k}^{[1]}\left(z_{k+1}\right)\right\| .
$$

We have to point out that relation (23) gives an approximation of the local error corresponding to the solution computed with the first order Split-Step method and consequently the size of the steps delivered by the adaptive step-size control method will be optimal for the first order SplitStep scheme. However, the solution computed by the Symmetric Split-Step method is a better approximation than the one computed by the first order Split-Step method and it is thus kept as the approximate solution. This is very common in such a situation and it is usually referred to as "local extrapolation". 


\subsection{The Embedded Split-Step method}

The computational sequence for one step of the Embedded Split-Step method can be understood as follows. For $k \in \mathbb{N}$, let $u_{k+1}^{[1]}$ denote the solution at grid point $z_{k+1}$ computed by the first order Split-Step scheme defined at step $k$ by (16)-(17)-(18) and let $u_{k+1}^{[2]}$ denote the solution at grid point $z_{k+1}$ computed by the Symmetric Split-Step method defined at step $k$ by (7)-(8)-(9). As mentioned before, the solution computed by the Symmetric Split-Step scheme is a better approximation than the one computed by the first order Split-Step scheme and it is kept as the approximate solution at each grid point. As a consequence, the initial condition in (7) and (16) is $A_{k-1}^{[2]}\left(z_{k}\right)$ and the 2 linear PDE problems are actually identical. It follows that the 2 problems (8) and (17) are also identical and the 2 Split-Step schemes only differ by (9) and (18).

The way $u_{k+1}^{[1]}$ and $u_{k+1}^{[2]}$ are obtained from $u_{k}^{[2]}$ (or actually their Fourier Transforms $\widehat{u}_{k+1}^{[1]}$ and $\widehat{u}_{k+1}^{[2]}$ from $\widehat{u}_{k}^{[2]}$ ) as well as the way the local error is estimated, can be summarized in the following computational sequence:

1: $\widehat{v}_{\frac{1}{2}}=\exp \left(\frac{h_{k}}{2} \widehat{d}_{v}\right) \times \widehat{u}_{k}^{[2]} \quad$ where $\widehat{d}_{v}$ is defined in (12)

2: $v_{\frac{1}{2}}=\mathcal{F}^{-1}\left(\hat{v}_{\frac{1}{2}}\right)$

3: $v_{1}= \begin{cases}v_{\frac{1}{2}} \exp \left(-\frac{\alpha}{2} h_{k}-\mathrm{i} \gamma\left|v_{\frac{1}{2}}\right|^{2} \frac{\mathrm{e}^{\alpha h_{k}-1}}{\alpha}\right) & \text { if } \alpha \neq 0 \\ v_{\frac{1}{2}} \exp \left(\mathrm{i} \gamma h_{k}\left|v_{\frac{1}{2}}\right|^{2}\right) & \text { if } \alpha=0\end{cases}$

4: $\widehat{v}_{1}=\mathcal{F}\left(v_{1}\right)$

5: $\widehat{u}_{k+1}^{[2]}=\exp \left(\frac{h_{k}}{2} \widehat{d}_{v}\right) \times \widehat{v}_{1}$

6: $\widehat{u}_{k+1}^{[1]}=\widehat{v}_{1}+\frac{h_{k}}{2} \widehat{d}_{v} \times \widehat{u}_{k}^{[2]}$

7: local error $=\left\|\widehat{u}_{k+1}^{[2]}-\widehat{u}_{k+1}^{[1]}\right\|_{0} \quad$ (from Parseval's theorem)

8: relative local error $=\left\|\widehat{u}_{k+1}^{[2]}-\widehat{u}_{k+1}^{[1]}\right\|_{0} /\left\|\widehat{u}_{k+1}^{[2]}\right\|_{0}$

When compared to the standard version of the Symmetric Split-Step method, the over-cost of the above computational procedure for delivering the local error estimate reduces to the computation of the term $\widehat{u}_{k+1}^{[1]}$ in line 6 . Since the value of $\frac{h_{k}}{2} \widehat{d}_{v}$ is required when implementing the Symmetric Split-Step scheme, the over-cost at each step is 1 multiplication and 1 addition times the number of sampling points in the frequency domain.

For step-size control, a tolerance "tol" is given as bound on the local error estimate. We consider the Symmetric Split-step method and the Split-Step method defined respectively by (7)(8)-(9) and (16)-(17)-(18), and we assume that the leading term in the asymptotic expansion (21) of the local error dominates the others for the current value of the step-size $h_{k}$. From (21) there exists $C \in \mathbb{R}^{+}$such that $\ell_{k+1}^{[1]}=C h_{k}^{2}$ and the optimal step-size $h_{\mathrm{opt}}$ is the one for which the local error estimate is the closest to the prescribed tolerance tol, i.e. $C h_{\mathrm{opt}}^{2}=$ tol. By eliminating the constant $C$ from these 2 relations we obtain $h_{\mathrm{opt}}=h_{k} \sqrt{\text { tol/err }}$ where err $=\ell_{k+1}^{[1]}$. For robustness the step-size control has to be designed in order to respond as smoothly as possible with real or apparent abrupt changes in behavior. As a consequence, the optimal step-size for $\widetilde{h}_{\text {opt }}$ for a prescribed tolerance tol is determined by a relation of the form $[19,20]$

$$
\widetilde{h}_{\text {opt }}=\max \left(\alpha_{2}, \min \left(\alpha_{1}, \alpha_{3} \sqrt{\frac{\text { tol }}{\text { err }}}\right)\right) h_{k}
$$

where $h_{k}$ is the current step-size, err is relative local error estimated from (23) and $\alpha_{1}, \alpha_{2}$ and $\alpha_{3}$ are additional tuning constant values. 


\section{Numerical comparisons on benchmark problems in optics}

We present a comparison of the Embedded Symmetric Split-Step (E3S) method presented in Section 4 with the Step-Doubling (SD) method [4] (without extrapolation) and with the Embedded split-step method based on the second-order Strang splitting and the first-order Lie-Trotter splitting (ESLT method) propound in [21] for solving 2 benchmark problems in optics.

\subsection{Soliton propagation}

When $\alpha=0$ and $\beta_{2}<0$, the NLSE (1) admits an exact solution known as the optical soliton [1, 2]. Namely, if the source term is given by $a_{0}: t \mapsto N_{s} /\left(\sqrt{\gamma L_{D}} \cosh \left(t / T_{0}\right)\right)$ where $N_{s}$ is the soliton order, $T_{0}$ is the pulse half-width and $L_{D}=-T_{0}^{2} / \beta_{2}$ is the dispersion length, then the solution to the NLSE at the soliton period $z_{p}=\frac{\pi}{2} L_{D}$ is given by

$$
\forall t \in \mathbb{R} \quad A\left(z_{p}, t\right)=\frac{N_{s}}{\sqrt{\gamma L_{D}}} \frac{\mathrm{e}^{\mathrm{i} z} N_{s}^{2} /\left(2 L_{D}\right)}{\cosh \left(t / T_{0}\right)} .
$$

We have considered a 3rd order soliton $\left(N_{s}=3\right)$ with the following physical parameters for the numerical experiment: $L=\frac{\pi}{2} L_{D}=19.80 \mathrm{~m}, \lambda=1550 \mathrm{~nm}, \gamma=4.3 \mathrm{~W}^{-1} \mathrm{~km}^{-1}, \beta_{2}=$ $-19.83 \mathrm{ps}^{2} \mathrm{~km}^{-1}, T_{0}=0.5 \mathrm{ps}$. The number of FFT points was set to $2^{14}$ and the time simulation windows to $180 \mathrm{ps}$. The initial step-size was $0.1 \mathrm{~m}$. The 3 tuning parameters in the step-size control formula (24) were set to $\alpha_{1}=2, \alpha_{2}=0.5$ and $\alpha_{3}=0.9$. Computations were done on an AMD A8 Personal Computer.

\begin{tabular}{|c|c|c|c|c|c|c|}
\hline Method & Tol. & CPU (s.) & Nb FFT & Nb steps (reject.) & Quad. Err. & Max. Err. \\
\hline \hline E3S & $10^{-3}$ & 10.9 & 834 & $416(2)$ & 0.004472 & 0.004526 \\
& $10^{-4}$ & 34.4 & 2618 & $1308(1)$ & 0.001006 & 0.001401 \\
\hline SD & $10^{-3}$ & 11.9 & 1016 & $338(36)$ & 0.011662 & 0.012961 \\
& $10^{-4}$ & 23.1 & 1922 & $640(0)$ & 0.002082 & 0.002251 \\
\hline ESLT & $10^{-3}$ & 26.3 & 1171 & $580(0)$ & 0.002477 & 0.002531 \\
& $10^{-4}$ & 83.4 & 3677 & $1838(0)$ & 0.0007714 & 0.001092 \\
\hline
\end{tabular}

Table 1: Comparison of the E3S, SD and ESLT methods for solving the NLSE for a 3rd order Soliton.

For comparison purposes we have summarized in Table 1 the main features of the 3 methods when applied for solving the normalized NLSE (1) with the above physical parameter values. We provide the CPU time in seconds, the number of FFT achieved, the number of computational steps with inside the brackets the number of steps rejected by the adaptive step-size control strategy and the quadratic relative error (relative error measured with the 2-norm) and the maximum relative error at the fiber end for tolerance values of $10^{-3}$ and $10^{-4}$. For a tolerance set to $10^{-3}$, one can observe that the E3S method requires a larger number of computational steps than the SD method but provides a result 2.5 times more accurate for a close computation time. Similar comments can be done in the case when the tolerance was set to $10^{-4}$ (excepted that the CPU time for the E3S method is 1.5 time larger than the one required by the SD method). One can also observe that the E3S method selects smaller step-sizes (average size of $0.0478 \mathrm{~m}$ for a tolerance $10^{-3}$ ) than the SD method (average size of $0.0655 \mathrm{~m}$ ). This behavior is confirmed in Fig. 1 where the evolution of the step-size along the fiber for the 2 methods is represented. This can be understood as follows. The adaptive step-size strategy in the E3S method estimates the local 


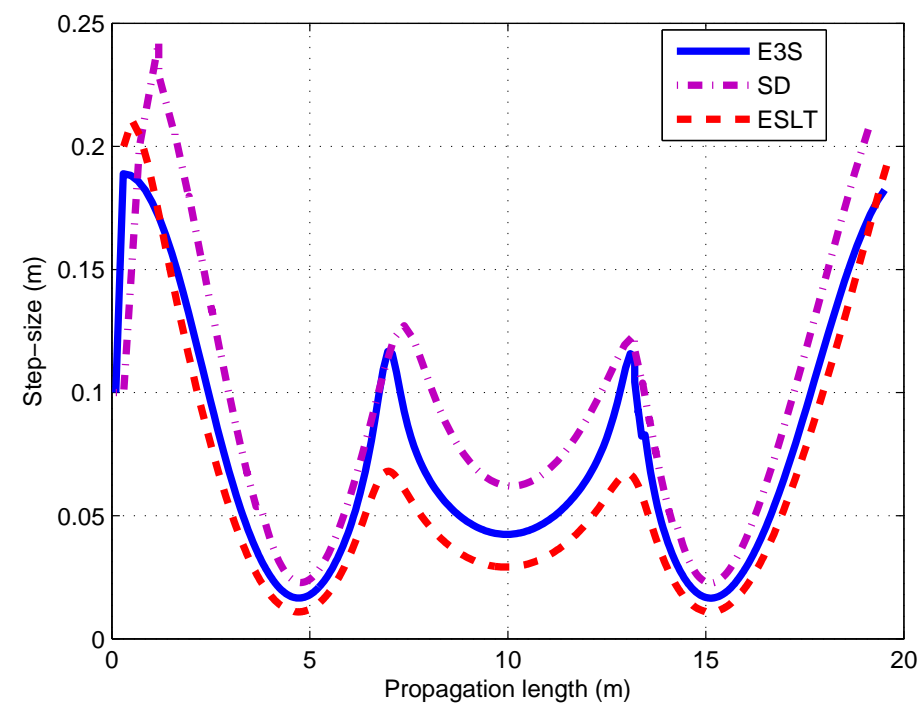

Figure 1: Evolution of the step-size along the fiber for the E3S, SD and ESLT methods for a 3rd order soliton with a tolerance set to $10^{-3}$.

error for the first order Split-Step scheme. This error is likely to be higher than the actual local error corresponding to the propagation of the solution of the second order Symmetric Split-Step scheme. This local error is more accurately estimated by the Step-Doubling method. The local error in the E3S method is therefore overestimated resulting in a selection of step-sizes smaller than actually required. This is the reason why the E3S method should not be used for too small tolerance values to be competitive compared to the SD method. However, the E3S method counterbalances this drawback by requiring less FFT evaluations per step and we can observe a gain in the computation time. Indeed, in order to obtain at the fiber end the same relative quadratic error of $0.45 \%$ corresponding to a tolerance $10^{-3}$ for the E3S method, the SD method should be used with a tolerance of $2.510^{-4}$. The CPU time is then $10.9 \mathrm{~s}$ for the E3S method versus $17.1 \mathrm{~s}$ for the SD method which represents a difference of approximatively $50 \%$. We have every reason to think that whenever the number of FFT nodes is large the E3S method will be much more efficient than the SD method (since the cost of each FFT will increase). This is confirmed by additional simulations: for instance when the number of FFT nodes is set to $2^{20}$ we obtain a CPU time of $676 \mathrm{~s}$ for the E3S method versus $1065 \mathrm{~s}$ for the SD method for a relative quadratic error of $0.45 \%$ at the fiber end and the same physical values as before. We refer to [22] for a practical application in optics requiring up to $2^{23}$ FFT nodes. Finally, when comparing the E3S method to the ESLT method one can see that the E3S method is much faster (around 2 times faster) than the ESLT method (actually the E3S method has been designed to meet this goal).

\subsection{Soliton collisions}

We present in this section numerical simulation results for the collision of 2 first order solitons [1]. It is known that when two neighboring solitons are launched with the same phase, they are initially attracted towards each other and then the two pulses periodically coalesce to form 


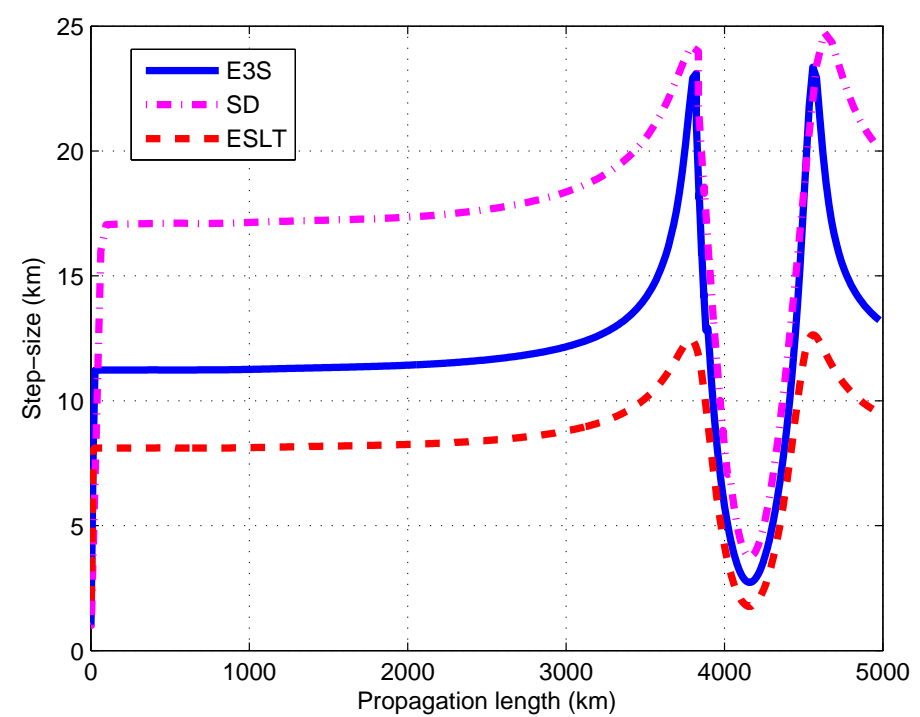

Figure 2: Evolution of the step-size along the fiber for the E3S, SD and ESLT methods for soliton collision.

one pulse and separate [23]. The source term was

$$
a_{0}: t \in \mathbb{R} \longmapsto \frac{1}{\sqrt{\gamma L_{D}}}\left(\frac{1}{\cosh \left(\left(t-T_{1}\right) / T_{0}\right)}+\frac{R \mathrm{e}^{\mathrm{i} \phi}}{\cosh \left(R\left(t+T_{1}\right) / T_{0}\right)}\right)
$$

where $T_{0}$ is the pulse half-width, $L_{D}=-T_{0}^{2} / \beta_{2}$ is the dispersion length, $R$ accounts for the relative amplitude, $\phi$ for the relative phase shift and $T_{1}$ for the initial separation time. The following physical parameters were taken for the numerical experiment: $L=5000 \mathrm{~km}, \lambda=1550 \mathrm{~nm}$, $\gamma=2.2 \mathrm{~W}^{-1} \mathrm{~km}^{-1}, \beta_{2}=-0.1 \mathrm{ps}^{2} \mathrm{~km}^{-1}, \alpha=0 \mathrm{~km}^{-1}, T_{0}=4 \mathrm{ps}, T_{1}=100 \mathrm{ps}, R=1$ and $\phi=0$. With these numerical values, the collision of the 2 solitons is predicted to happen at a distance of $4161 \mathrm{~km}$ [1]. The simulation time windows was $400 \mathrm{ps}$ and the number of FFT nodes was $2^{14}$. The initial step-size was set to $1 \mathrm{~km}$ and the tolerance to $10^{-3}$.

For comparison purposes we have summarized in Table 2 the main features of the 3 methods when applied for solving the NLSE with the above physical parameter values. The relative global error at the fiber end has been computed by comparison to a reference solution obtained with a constant step-size of $0.1 \mathrm{~km}$. Fig. 2 depicts the evolution of the step-size along the fiber. The same observations made in the previous example are also valid here.

\begin{tabular}{|c|c|c|c|c|c|}
\hline Method & CPU time (s.) & Nb FFT & Nb steps (rejected) & Quad. Err. & Sup. Err. \\
\hline \hline E3S & 12.2 & 974 & $486(4)$ & 0.014715 & 0.014978 \\
\hline SD & 11.6 & 1064 & $354(32)$ & 0.031737 & 0.032481 \\
\hline ESLT & 30.6 & 1361 & $680(0)$ & 0.007371 & 0.007494 \\
\hline
\end{tabular}

Table 2: Comparison of the E3S, SD and ESLT methods for solitons collision with a tolerance set to $10^{-3}$.

In order to obtain at the fiber end the same relative quadratic error of $1.47 \%$ corresponding 
to a tolerance $10^{-3}$ for the E3S method, the SD and the ESLT methods should be used with a tolerance of $310^{-4}$ and $210^{-3}$ respectively. For the SD method the CPU time is then $17.0 \mathrm{~s}$ and 512 steps are required whereas for the ESLT method the CPU time is $21.9 \mathrm{~s}$ and 481 steps are required.

\section{Conclusion}

We have presented a new way of estimating the local error for adaptive step-size control purposes when solving the nonlinear Schrödinger equation (NLSE) by using the Symmetric SplitStep method. Compared to the Step-Doubling approach where the 2 approximated solutions are obtained by solving the NLSE by the Symmetric Split-Step method with 2 different step-sizes (the finest one being half the coarse one) resulting in a computational over-cost of $50 \%$, our approach gives a coarse approximated solution at low cost by a first order Split-Step scheme designed to be fully embedded into the Symmetric Split-Step method. The numerical investigations we have conducted show that this way of estimating the local error for adaptive step-size purposes is valuable compared to the Step-Doubling approach when the computational cost of the Fourier Transform is significant (which is likely to occur in optics e.g. when the self-phase modulation induces a large spectral broadening or in the presence of Raman induced frequency shift) since the Step-Doubling approach increases by $50 \%$ the number of Fourier Transforms to be achieved compared to the Symmetric Split-Step method without adaptive step-size control. Numerical experiments have shown that a drawback of the propound method is that it slightly overestimates the local error resulting in a selection of step-sizes smaller than optimal (the reason is that the local error for the Symmetric Split-Step method is estimated from a first order Split-Step scheme). The various parameters in the step-size selection formula could however be empirically tuned to take into account this particularity. Alternatively higher order embedded Split-Step scheme could be used. Compared to the embedded split-step pair based on Strang and Lie-Trotter splitting formulae proposed in [21] for general evolution problems, our Embedded split-step method designed specifically for solving the NLSE (1) exhibits better numerical performances.

\section{References}

[1] G. Agrawal. Nonlinear fiber optics. Academic Press, 3rd edition, 2001.

[2] K. Okamoto. Fundamentals of Optical Waveguides. Optics and Photonics. Elsevier, 2006.

[3] B. Hermansson, D. Yevick, and A. Friberg. Optical coherence calculations with the Split-Step fast Fourier transform method. Appl. Opt., 25(16):2645-2647, 1986.

[4] O.V. Sinkin, R. Holzlöhner, J. Zweck, and C.R. Menyuk. Optimization of the Split-Step Fourier method in modeling optical-fiber communications systems. J. Lightwave Technol., 21(1):61, 2003.

[5] N. Feng, G. Zhou, and W. Huang. An efficient Split-Step time-domain beam-propagation method for modeling of optical waveguide devices. J. Lightwave Technol., 23(6):2186, 2005.

[6] Z. Toroker and M. Horowitz. Optimized Split-Step method for modeling nonlinear pulse propagation in fiber Bragg gratings. J. Opt. Soc. Am. B, 25(3):448-457, 2008.

[7] R. Deiterding, R. Glowinski, H. Oliver, and S. Poole. A reliable Split-Step Fourier method for the propagation equation of ultra-fast pulses in single-mode optical fibers. J. Lightwave Technol., 31(12):2008-2017, 2013.

[8] C. Besse, B. Bidégaray, and S. Descombes. Order estimates in time of splitting methods for the nonlinear Schrödinger equation. SIAM J. Numer. Anal., 40(1):26-40, 2002.

[9] V. Zharnitsky. Averaging for Split-Step scheme. Nonlinearity, 16(4):1359-1366, 2003

[10] C. Lubich. On splitting methods for Schrödinger-Poisson and cubic nonlinear Schrödinger equations. Math. Comp., $77: 2141-2153,2008$ 
[11] D. Gilbarg and N. Trudinger. Elliptic Partial Differential Equations of Second Order. Springer-Verlag, Berlin, 1977.

[12] R. Carles. Semi-classical analysis for nonlinear Schrödinger equations. World Scientific, 2008

[13] T. Cazenave. Semilinear Schrodinger Equations. Courant Lecture Notes in Mathematics, AMS, New York, 2003.

[14] S. Balac, A. Fernandez, F. Mahé, F. Méhats, and R. Texier-Picard. The Interaction Picture method for solving the nonlinear Schrödinger equation in optics. Technical report, 2013. http://hal.archives-ouvertes.fr/ hal-00850518/.

[15] A. Rieznik, T. Tolisano, F. A. Callegari, D. Grosz, and H. Fragnito. Uncertainty relation for the optimization of optical-fiber transmission systems simulations. Opt. Express, 13(10):3822-3834, May 2005.

[16] T. Meirelles, A. Rieznik, and H. Fragnito. Study on a new Split-Step Fourier algorithm for optical fiber transmission systems simulations. In Microwave and Optoelectronics, 2005 SBMO/IEEE MTT-S International Conference on, pages 100-102, 2005.

[17] A. Heidt. Efficient adaptive step size method for the simulation of supercontinuum generation in optical fibers. $J$. Lightwave Technol., 27(18):3984-3991, 2009.

[18] L. Shampine. Local error estimation by doubling. Computing, 34:179-190, 1985.

[19] J.C. Butcher. Numerical methods for ordinary differential equations. John Wiley and Sons, 2008

[20] E. Hairer, S.P. Norsett, and G. Wanner. Solving ordinary differential equations I: nonstiff problems. Springer-Verlag New York, 1993.

[21] O. Koch, C. Neuhauser, and M. Thalhammer. Embedded Split-Step formulae for the time integration of nonlinear evolution equations. Appl. Numer. Math., 62:14-24, 2013.

[22] A. Fernandez, S. Balac, A. Mugnier, F. Mahé, R. Texier-Picard, T. Chartier, and D. Pureur. Numerical simulation of incoherent optical wave propagation in nonlinear fibers. Eur. Phys. J. - Appl. Phys., 64:24506/1-11, 2013.

[23] C. Desem and P.L. Chu. Reducing soliton interaction in single-mode optical fibres. Optoelectronics, IEE Proceedings $J, 134(3): 145-151,1987$. 